\title{
New N-Capture Element Abundance Determinations in an $r$-Process Enriched Star
}

\author{
John J. Cowan ${ }^{* t}$ \\ HLD Dept. of Physics \& Astronomy, University of Oklahoma, Norman, OK 73019 \\ E-mail: cowan@nhn.ou.edu
}

Ian U. Roederer

Carnegie Observatories, 813 Santa Barbara Street, Pasadena, CA 91101

E-mail: iur@obs.carnegiescience.edu

\section{Christopher Sneden}

Department of Astronomy, University of Texas at Austin, Austin, TX 78712

E-mail: chris@verdi.utexas.edu

\section{James E. Lawler}

Department of Physics, University of Wisconsin, Madison, WI 53706

E-mail: jelawler@wisc.edu

We report on new abundance determinations for the neutron-capture elements Cd I ( $\mathrm{Z}=48)$, Lu II $(\mathrm{Z}=71)$ and Os II $(\mathrm{Z}=76)$ in the $r$-process enriched star BD +17 3248. These abundances are derived from an ultraviolet spectrum obtained with the Space Telescope Imaging Spectrograph on the Hubble Space Telescope. These are the first detections of these elements in metal-poor $r$-process enriched halo stars. In addition to the HST observations we have obtained new abundance measurements of the elements Mo I, Ru I and Rh I, based upon High Resolution Echelle Spectrograph observations with Keck. Combining these and previous observations, we have now detected $32 n$-capture elements in $\mathrm{BD}+173248$ - this is the most of any metal-poor halo star to date. The lighter $n$-capture elements $(38 \leq \mathrm{Z} \leq 48$ ) appear to show a pronounced odd-even effect. New Hf I abundances from transitions in the UV were also derived and they were lower than previous values based upon optical transitions. The new Hf abundance agrees better with the scaled Solar system $r$-process distribution. We also derive an age for this star based upon the Th II/Os II chronometer, which is in better agreement with the age derived from other chronometers than the age derived using Th II/Os I.

11th Symposium on Nuclei in the Cosmos, NIC XI

July 19-23, 2010

Heidelberg, Germany

\footnotetext{
* Speaker.

${ }^{\dagger}$ Funding for this project has been generously provided by NSF.
} 


\section{Introduction}

Abundance determinations of the neutron-capture (aka heavy) elements in metal-poor halo stars are fundamentally important to our understanding of the early history of the Galaxy and the Universe. These elements are synthesized in either the slow $(s)$ or rapid $(r)$ neutron-capture process in generations of stars preceding the halo stars[1]. In the last several decades increasing numbers of elements have been detected in an ever larger number of stars. Furthermore, these abundance determinations have become much more accurate as a result of better (and more) high-resolution observations that have taken advantage of increasingly precise experimental atomic physics data[2, 3]. This in turn has provided a more complete understanding of the nature of the earliest nucleosynthetic processes and clues to the identities of the first generations of stars in the Galaxy and the Universe.

\section{New Elemental Abundance Detections}

Recently Roederer et al.[4] have employed the Hubble Space Telescope (HST) to detect CD I $(\mathrm{Z}=48), \mathrm{Lu} \mathrm{II}(\mathrm{Z}=71)$ and Os II $(\mathrm{Z}=76)$ in the metal-poor $r$-process enriched halo star $\mathrm{BD}+17$ 3248. We show in Figure 1 (left) the spectra of three well-studied halo stars: BD+17 3248, HD 122563 and HD 115444 in the region around an Os II and a Cd I line. There are clear detections of Os II in BD+17 3248 and HD 115444, but not in the bright, but $r$-process poor, star HD 122563. We see in the lower panel that $\mathrm{Cd} \mathrm{I}$ is detected in all three stars. In the right side of Figure 1 we illustrate the fit of synthetic spectra to the actual spectra in BD+17 3248 and HD 122563. In the top panel the best fits (bold lines) are shown for Os II, Cd I and Lu II lines in BD+17 3248, with the corresponding lines for HD 122563 shown in the bottom panel. The plots also illustrate (with a thin line) a synthesis with the species of interest removed and variations $( \pm 0.3 \mathrm{dex})$ around the best fit (dotted lines). These syntheses demonstrate the detection of Os II, Cd I and Lu II in BD+17 3248 and Cd I and Lu II (but not Os II) in HD 122563. These UV observations were the first detections of these neutron-capture species in metal-poor stars enriched by the $r$-process.

\section{Discussion}

The new observations of [4] indicate a clear contrast between ( $r$-poor) HD 122563 and ( $r$-rich) $\mathrm{BD}+17$ 3248. In the case of HD 122563 there is a dramatic fall-off in the elemental abundances as a function of atomic number with no detection of the heavier elements like Hf and Os $([5,4]$. The newly determined $\mathrm{Cd}$ abundance reaffirms this downward trend between the first and second $r$-process peaks, while the Os upper limit is not strong enough to exclude a scaled solar system $r$ process pattern. This abundance trend can be interpreted as an incomplete $r$-process in the material that was injected into the gas that formed HD 122563. This suggests that there were insufficient neutron fluxes (in the astrophysical synthesis site) to move far from the valley of $\beta$-stability or to reproduce the total solar system $r$-process curve - in other words the $r$-process path was not in the correct place to be able to synthesize the heaviest elements and to reproduce the (second and third) $r$-process abundance peaks (see [6] for further discussion of the $r$-process.) 


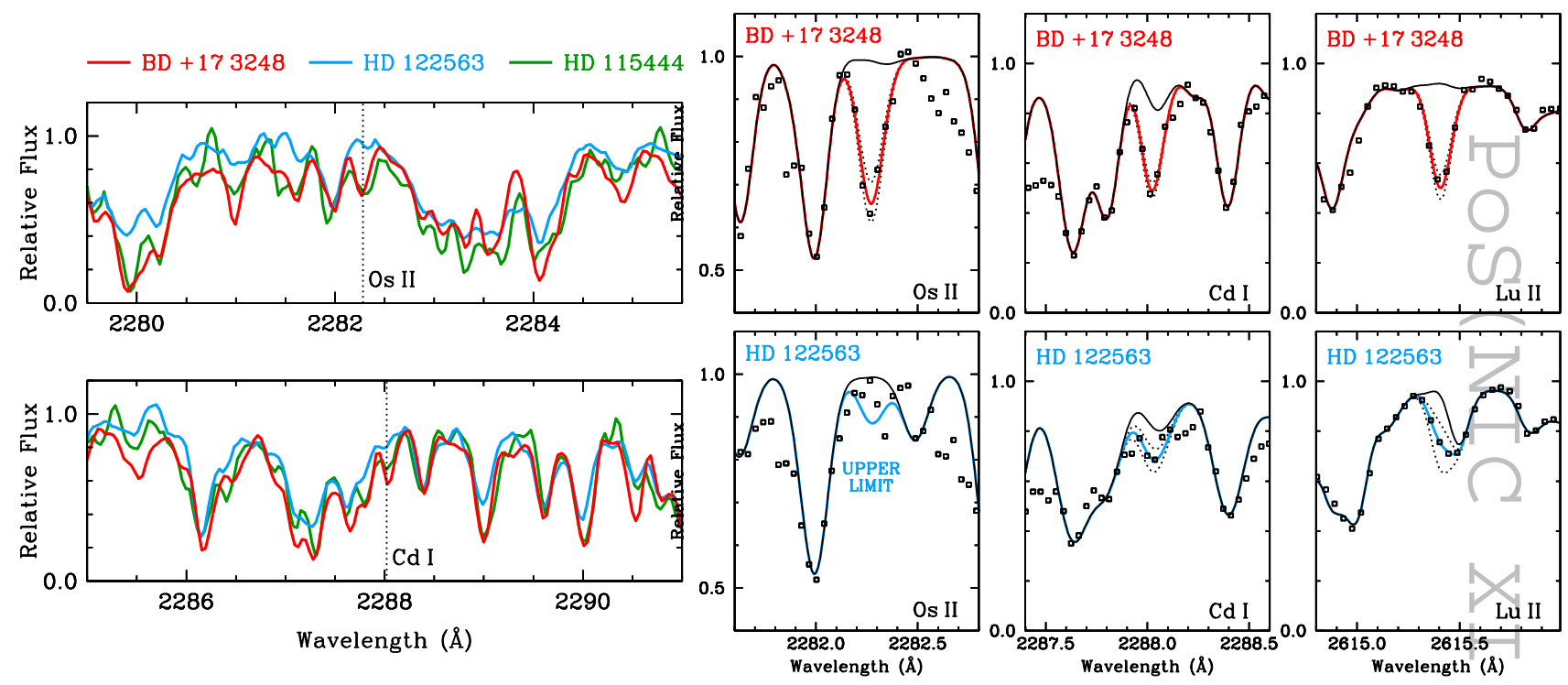

Figure 1: (left) Spectra of BD+17 3248, HD 122563 and HD 115444 showing Os II and Cd I lines. (right) Synthetic spectral fits to the spectra of BD+17 3248 and HD 122563. The bold lines indicate the best fit, dotted lines indicate \pm 0.3 dex from the best fit, and the thin line indicates a non-detection. (Both figures after [4].)

To supplement the new HST observations of BD+17 3248 additional (High Resolution Echelle Spectrograph) Keck data was analyzed by [4]. That analysis provided new abundance determinations for the $n$-capture species Mo I, Ru I and Rh I in this star. These bring to 32 (not counting $\mathrm{Ge}$ ) the number of $n$-capture elemental abundance detections in BD+17 3248. This is the most of any metal-poor, $r$-process-rich halo star to date - beating CS 22892-052 by one. We note that HST observations of very low-metallicity halo stars ([7]) indicate that Ge appears to scale with iron production. This has led to suggestions of neutrino (i.e., $v$-p) processes in supernovae as the synthesis mechanism for this element ([8]), at least early in the history of the Galaxy. At later Galactic times (and higher metallicities) the $s$-process makes significant contributions to the production of this element ([1]).

We show the total $n$-capture elemental abundance distribution for BD+17 3248 in Figure 2. These abundances are compiled from the observations of $[9,7,2,10,4]$. Abundance comparisons with solar system $r$-process only predictions $([11,1])$ are also illustrated in the figure. It is clear that for the heaviest $n$-capture elements, (i.e., $\mathrm{Ba}$ and above), the abundance distribution in $\mathrm{BD}+17$ 3248 is an excellent fit to the solar system $r$-process abundances from either [11] or from [1]. This includes in particular a new abundance determination for Hf. Previous observations of optical lines for this element indicated an abundance above the solar system $r$-process line by approximately $\log \varepsilon=0.15-0.25$ ([12, 10, 2]). The new NUV abundance determination for Hf II in BD+17 3248 
indicates a lower value, and one that is now in good agreement with the solar system curve.

Examination of Figure 2 indicates that the lighter $n$-capture elemental abundances are not in close agreement with the solar system $r$-process curve. This has been seen in other stars as well, for example CS 22892-052 ([13]). There have been many suggestions for explaining the synthesis of these elements from Sr-Ba, including the "weak $r$-process" ([14]), a light element primary process, LEPP $([15,16])$ the $v$-p process $([8])$, or a combination of several of these processes that might also include the main $r$-process, charged particle processes or the weak $s$-process ([17]). Recently, dynamical calculations simulating the conditions in the high-entropy winds (HEW) from a core collapse supernova were (encouragingly) able to reproduce the $\mathrm{Sr}$-Pd elemental abundances in BD+17 3248 [18]. But further calculations and comparisons will be needed to reproduce the evenodd effect of the cadmium and silver abundances.

The newly determined Os II abundance value in BD+17 3248 can be utilized as part of the chronometric pair Th II/Os II to provide radioactive age estimates. Assuming initial production ratios from [14], the age range derived (7.9-12.3 Gyr), while still large, is more consistent with other stellar chronometric age estimates ([10]) than that based upon the ratio of Th II/Os I. Reducing the Os II abundance uncertainty could lead to more precise age estimates for this star.

Our new HST abundance determinations of Cd I, Lu II and Os II in BD+17 3248 are the first in $r$-process enriched halo stars and have expanded the total number of $n$-capture elements detected to 32 - the most of any of these stars to date. These latest elemental determinations are providing new clues and information about the synthesis processes and the first generations of stars that occurred early in the history of the Galaxy.

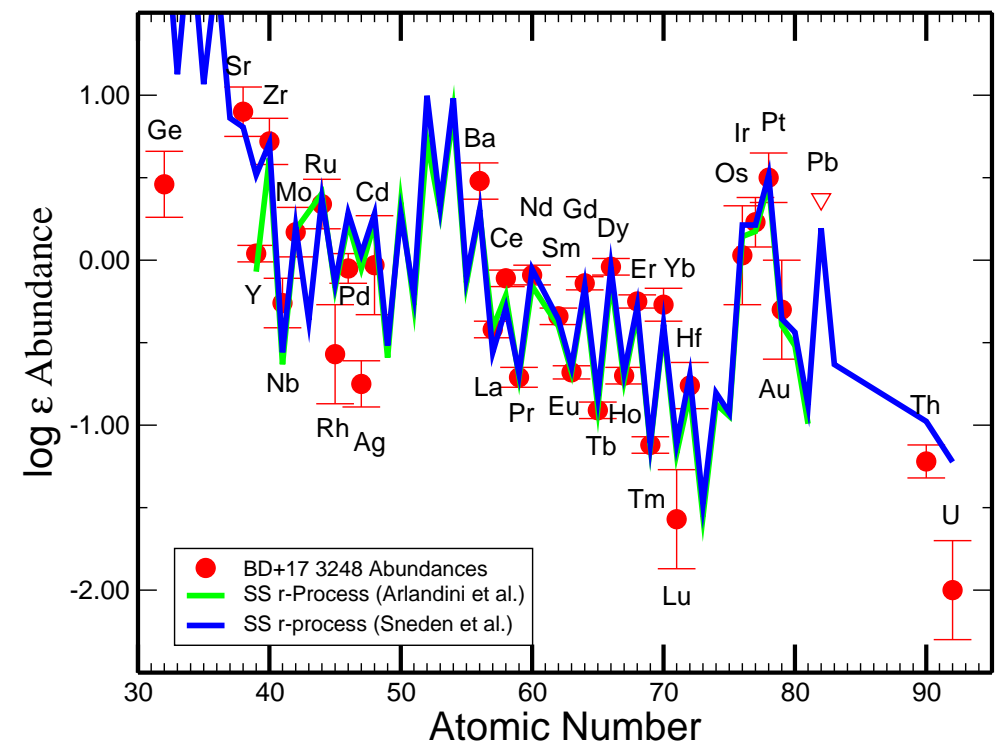

Figure 2: N-capture element abundances in BD+17 $3248[9,7,2,10,4]$ compared with two solar system $r$-process only predictions $[1,11]$. 


\section{References}

[1] Sneden, C., Cowan, J. J., \& Gallino, R. Neutron-capture elements in the early galaxy. Annual Reviews of Astronomy \& Astrophysics 46, 241-288 (2008).

[2] Sneden, C., Lawler, J. E., Cowan, J. J., Ivans, I. I., \& Den Hartog, E. A. New rare earth element abundance distributions for the Sun and five $r$-process-rich very metal-poor stars. Astrophys. J. Supp. 182, 80-96 (2009).

[3] Lawler, J. E., Sneden, C., Cowan, J. J., Ivans, I. I., \& Den Hartog, E. A. New rare earth element abundance distributions for the Sun and five $r$-process-rich very metal-poor stars. Astrophys. J. Supp. 182, 51-79 (2009).

[4] Roederer, I. U., Sneden, C., Lawler, J. E., \& Cowan, J. J. New abundance determinations of Cadmium, Lutetium and Osmium in the $r$-process enriched star BD+17 3248. Astrophys. J. 714, L123-L127 (2010).

[5] Honda, S., Aoki, W., Ishimaru, Y., Wanajo, S., \& Ryan, S. G. Neutron-capture elements in the very metal poor star HD 122563, Astrophys. J. 643, 1180-1189 (2006).

[6] Cowan, J. J. \& Thielemann, F.-K. R-process nucleosynthesis in supernovae. Phys. Today. 57, 47-53 (2004).

[7] Cowan, J. J., et al. Hubble Space Telescope observations of heavy elements in metal-poor galactic halo stars. Astrophys. J. 627, 238-250 (2005).

[8] Frölich, C., et al. Neutrino-induced nucleosynthesis of A $>64$ nuclei The vp-process. astro-ph/0511376, Phys. Rev. Lett. 96, 142502-1-142502-4 (2005).

[9] Cowan, J. J., et al. The chemical composition and age of the metal-poor halo star BD $+17^{\circ} 3248$. Astrophys. J. 572, 861-879 (2002).

[10] Roederer, I. U., et al. The end of nucleosynthesis: production of lead and thorium in the early galaxy Astrophys. J. 698, 1963-1980 (2009).

[11] Arlandini, C., et al. Neutron capture in low-mass asymptotic giant branch stars: cross sections and abundance signatures Astrophys. J. 525, 886-900 (1999).

[12] Lawler, J. E., Den Hartog, E. A., Labby, Z. E.,Sneden, C., Cowan, J. J. \& Ivans, I. I. Hf transition probabilities and abundances. Astrophys. J. Supp., 169, 120-136 (2007).

[13] Sneden, C., et al. The extremely metal-poor neutron capture-rich star CS 22892-052: A comprehensive abundance analysis. Astrophys. J. 591, 936-953 (2003).

[14] Kratz, K.-L., et al. Explorations of the $r$-process: comparisons between calculations and observations of low metallicity stars. Astrophys. J., 662, 39-52 (2007).

[15] Travaglio, C., Gallino, R., Arnone, R., Cowan, J. J., Jordan, F. \& Sneden, C. Galactic evolution of Sr, Y and Zr: a multiplicity of nucleosynthetic processes. Astrophy. J. 601, 864-884 (2004).

[16] Montes, F., et al. Nucleosynthesis in the early galaxy. Astrophy. J. 671, 1685-1695 (2007).

[17] Roederer, I. U., et al. The ubiquity of the rapid neutron-capture process. arXiv:1009.4496, Astrophys. $J$. in press (2010).

[18] Farouqi, K., et al. Nucleosynthesis modes in the high-entropy-wind of type II supernovae: comparison of calculations with halo-star observations Astrophys. J. 694, L49-L53 (2009). 